\title{
EXPERIENCIA DEL TRATAMIENTO DE ESPONDILOLISTESIS LUMBAR DEGENERATIVA DE UN SOLO SEGMENTO CON ESPACIADOR INTERESPINOSO
}

\author{
EXPERIÊNCIA NO TRATAMENTO DE ESPONDILOLISTESE LOMBAR DEGENERATIVA \\ DE UM SÓ SEGMENTO POR MEIO DE ESPAÇADOR INTERESPINHAL \\ EXPERIENCE IN THE TREATMENT OF DEGENERATIVE LUMBAR SPONDYLOLISTHESIS \\ OF JUST ONE SEGMENT USING IN INTERSPINOUS IMPLANT
}

Rodrigo Félix Garza', Juan Lagarda Cuevas ${ }^{1}$, Mauro Francisco Chavez Maqueda', Guillermo Iván Ladewig Bernaldez ${ }^{1}$

\begin{abstract}
RESUMEN
Objetivo: Evaluar a un año el resultado del uso de espaciadores dinámicos en listésis grado I de Meyerding utilizando la escala de incapacidad de Oswestry. Métodos: Se revisa el historial electrónico y radiográfico de los pacientes según criterios en el período de enero 2008 a diciembre 2010, con el propósito de realizar un estudio de cohortes, retrospectivo, longitudinal y observacional. Resultados: El Oswestry prequirúrgico fue de 3.4\% leve, 55.2\% moderado y $41.4 \%$ severo; mientras que el posquirúrgico fue de $79.3 \%$ leve y $20.7 \%$ moderado. La cirugía realizada más común fue exploración y liberación con un 72.4\%, presentando discectomía únicamente el 27.6\%. Los pacientes presentaron dolor irradiado a miembro pélvico derecho en el 37.9\%, miembro pélvico izquierdo $44.8 \%$ y a ambos miembros pélvicos en un $17.2 \%$. Se presentó dolor posquirúrgico irradiado a miembro pélvico únicamente en el $2.4 \%$ siendo que el $100 \%$ de los casos presentaron algún tipo de dolor irradiado. Se utilizó espaciador DIAM en 79.3\% y Wallis en 20.7\% Conclusiones: El tratamiento con espaciador interespinoso presenta un bajo índice de reintervención y por lo menos a un año presenta mejoría significativa en el índice de incapacidad.
\end{abstract}

Descriptores: Columna vertebral; Dolor de la región lumbar; Espondilolistesis; Fijadores internos.

\section{RESUMO}

Objetivo: Avaliar o resultado em ano do uso de espaçadores dinâmicos em listese de grau I de Meyerding, utilizando a escala de incapacidade de Oswestry. Métodos: Foi revisado o prontuário eletrônico e as radiografias dos pacientes, de acordo com os critérios, no período de janeiro de 2008 a dezembro de 2010, visando realizar um estudo de coortes, retrospectivo, longitudinal e observacional. Resultados: O índice de Oswestry pré-cirúrgico foi de 3,4\% leves, 55,2\% moderados e 41,4\% graves, enquanto o pós-cirúrgico foi de $79,3 \%$ leves e 20,7\% moderados. A cirurgia mais realizada foi exploração e liberação, em 72,4\%, sendo que 27,6\% apresentaram apenas discectomia. Os pacientes tinham dor irradiada para o membro inferior direito em $37.9 \%$, para o membro inferior esquerdo em 44,8\% e para ambos os membros inferiores, em 17,2\%. Verificou-se dor pós-operatória irradiada para o membro inferior só em $2,4 \%$, sendo que $100 \%$ dos casos tinham algum tipo de dor irradiada. Foi empregado o espaçador DIAM em 79.3\% e o Wallis em 20.7\% Conclusões: O tratamento com espaçador interespinhoso apresenta baixo índice de re-intervenção e pelo menos em um ano, apresenta melhora significante do índice de incapacidade.

Descritores: Coluna vertebral; Dor lombar; Espondilolistese; Fixadores internos.

\begin{abstract}
Objective: To evaluate one-year results of using dynamic spacers in listhesis grade I of Meyerding using Oswestry Disability Index. Methods: The disability scale review was based in the electronic and radiographic history of patients according to criteria in the period from January 2008 to December 2010, in order to conduct a cohort study, retrospective, longitudinal, and observational. Results: The Oswestry index before surgery was $3.4 \%$ mild, $55.2 \%$ moderate and severe $41.4 \%$, while the postoperative was $79.3 \%$ mild and $20.7 \%$ moderate. The most common surgery performed was exploration and release in $72.4 \%$, and only $27.6 \%$ had discectomy. Patients had pain radiating to right lower limb in $37.9 \%$, to the left lower limb in $44.8 \%$ and to both lower extremities in $17.2 \%$. Pain radiating to the lower limb after surgery was found only in $2.4 \%$, and $100 \%$ of the cases had some type of radiating pain. The DIAM interspinous implant was used in $79.3 \%$, and the Wallis in 20.7\%. Conclusions: Treatment with interspinous spacer has a low rate of reoperation and at least during one year presented significant improvement in the rate of disability.
\end{abstract}

Keywords: Spine; Low back pain; Spondylolisthesis; Internal implants.

\section{INTRODUCCIÓN}

El término de espondilolistesis degenerativa fue acuñado por New$\operatorname{man}^{1}$ en 1963 y se define como el deslizamiento hacia adelante de una vértebra inmediatamente inferior, sin lisis ístmica. Dicho fenómeno se observa predominantemente en personas mayores de 40 años y con un predominio de 4:1 del sexo femenino, teniendo una prevalencia $7.5 \%$ en hombres y del $16.7 \%$ y $28 \%$ en mujeres nulíparas y multíparas respectivamente en paciente con lumbalgia mayores a 50 años. ${ }^{2}$

1. Hospital Central Sur de Alta Especialidad PEMEX, Distrito Federal, México.

2. Hospital de Especialidades CMN siglo XXI, Distrito Federal, México.

Trabajo realizado en Hospital Central Sur de Alta Especialidad Petróleos Mexicanos, Distrito Federal, México.

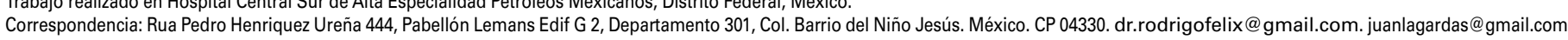


El segmento que con mayor frecuencia se ve afectado es L4-L5 (85\% de los casos) seguido de L3-L4 e involucro raro de L5-S1, se ha postulado que la fisiopatología es una combinación de degeneración facetaria y discal. (Figura 1)

Los pacientes generalmente cursan con claudicación neurogénica así como con síntomas de estenosis espinal y dolor lumbar previo a la aparición de los síntomas en miembros pélvicos, aunque el tratamiento conservador puede disminuir de manera importante los síntomas, la cirugía provee de alivio más rápido y duradero.,4

Si bien en casos de estenosis pura, puede optarse por realizar únicamente descompresión limitada (laminoforaminotomía o descompresión interlaminar) se ha visto que la laminectomía y fusión lumbar de manera conjunta presentan mejores resultados ${ }^{5,6}$ en especial a largo plazo.

En pacientes con comorbilidades importantes, así como con osteoporosis se prefiere únicamente la fusión sin fijación, ${ }^{7}$ aun teniendo en cuenta estos parámetros, el "gold standard" para manejo de lumbalgia crónica es la fusión dorsal.

En 1931, Meyerding 8 describe la subluxación lumbar en porcentaje, asignándole a la misma diferentes grados, el sistema clasifica la severidad basándose en la proyección lateral de columna lumbar y mide la distancia del borde posterior de la vértebra superior al borde posterior de la vértebra inferior.

Así pues menciona que:

Grado I: $<25 \%$

Grado II: $25-30 \%$

Grado III: $50-75 \%$

Grado IV: $75-100 \%$

Mayor a $100 \%$ se le denomina espondiloptosis. ${ }^{9,10}$

La clasificación del grado de espondilolistésis de Mayerding sigue siendo la más ampliamente utilizada para valorar radiografías así como la TAC. ${ }^{11}$

El dolor lumbar a menudo implica una carga anormal más que un movimiento anómalo como causa del dolor, así encontramos que muchos pacientes se quejan dolor relacionado a la postura como síntoma predominante, en un estudio in vivo, Mulholland e Sengupta ${ }^{12}$, McNally et al. ${ }^{13}$ demostró que más que los niveles absolutos de la carga lo que genera el dolor son los patrones de la carga.

Este hecho correlaciona de manera clínica al haber poca correspondencia entre los grados de degeneración y el dolor lumbar entre individuos. ${ }^{12,13}$

En las últimas 2 décadas la justificación predominante para la fusión dorsal ha sido la inestabilidad. ${ }^{11-13}$

El término de inestabilidad hace referencia a un movimiento anormal bajo una carga fisiológica y aunque se ha tratado de relacionar con el dolor lumbar no se ha encontrado una relación clara hasta el momento. ${ }^{12,13}$

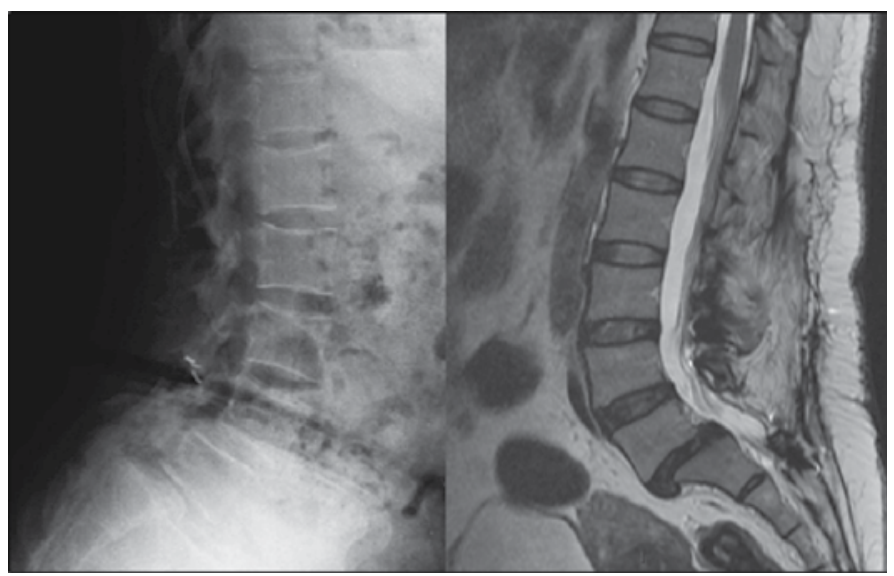

Figura 1. Radiografía Simple e Imagen por Resonancia Magnética con una listesis degenerativa Grado I de Meyerding.
Debido a estos puntos antes mencionados, se ha planteado que la fusión podría no ser la mejor opción y que una estabilización dinámica sería más apropiada para aliviar el dolor, además se tiene que tomar en cuenta la complicaciones de la fusión dorsal como lo son cambios osteoartríticos facetarios, ${ }^{14}$ fracturas por fatiga, fallas en la instrumentación, síndrome del segmento adyacente y pérdida de la lordosis. ${ }^{15}$

La estabilización dinámica ha sido definida como: "un sistema que altera de manera favorable el movimiento y la transmisión de la carga de un segmento espinal móvil, sin la intensión de fusionar el segmento". ${ }^{14,15}$

En general, los dispositivos interespinosos, (Figura 2) flotan entre ambas espinosas y actúan como espaciador entre ambos procesos espinosos, biomecánicamente su presencia limita la extensión sin efecto en la flexión, rotación axial o flexión lateral. ${ }^{15,16}$

Los dispositivos dinámicos que se encuentran disponibles hasta el momento se colocan por vía posterior y por consideraciones anatómicas del proceso espinoso de S1 no se recomienda su uso para el segmento L5-S1. ${ }^{17}$

Dentro de los espaciadores interespinosos existen, entre otros, los que son en forma una cuña de silicón en forma de $\mathrm{X}$ cubierto de poliéster, dos cordones de seguridad están adheridos a la cuña, la colocación de dicho implante requiere de una incisión de 4-5 $\mathrm{cm}$ longitudinales y puede realizarse con la preservación de los ligamentos supra e interespinosos, ó sin ellos. ${ }^{18}$

Se ha sugerido que es capaz de compartir la carga y controlar el movimiento, ya que parte de la carga es distribuida hacia posterior y compartida con el disco por el dispositivo, convirtiéndose en un sistema que soporta la carga. ${ }^{19}$

También existen implantes para estabilización no rígida de un segmento lumbar de segunda generación, este bloqueador interespinoso que se encuentra hecho de PEEK (polietererercetona), constituye un sistema "flotante", esto se debe a que no tiene fijación permanente en el hueso vertebral para evitar riesgo de aflojamiento ocasiona un aumento en la rigidez de los segmentos no estables. ${ }^{20-22}$

La espondilolistésis es un problema común que causa discapacidad física importante en pacientes en edad productiva, los métodos que por lo regular se implementan para el tratamiento de dicho problema requieren de la fusión dorsal ya sea por medio de fijación transpedicular, fusión intersomática o artrodesis posterolateral.

El uso del separador interespinoso tipo DIAM permite una cirugía más simple sin necesidad de tanto daño a estructuras anatómicas que permite una rehabilitación más temprana y ausencia de dolor persistente.

La escala de incapacidad de Oswestry, ODI por sus siglas en inglés, fue desarrollada por Fairbank et al. ${ }^{23}$ en 1980 para indicar hasta qué grado el nivel funcional de una persona se ve restringido por la incapacidad, desde su publicación el ODI ha sido una

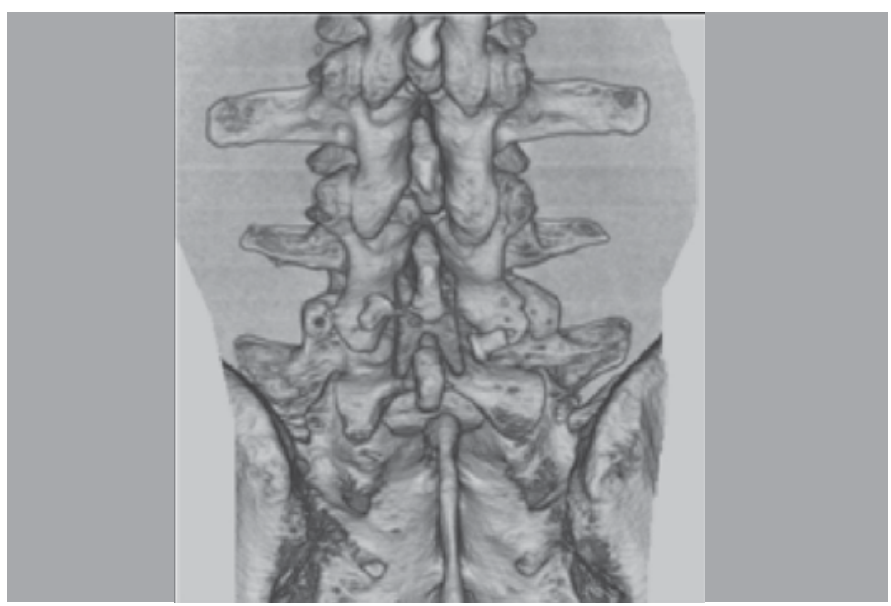

Figura 2. Reconstrucción tridimensional de espaciador Interespinoso L4-L5. 
herramienta mundialmente utilizada y continúa siendo válida como método pronóstico en los padecimientos de columna. ${ }^{24}$

Si bien, se conocen por lo menos tres variantes de la escala de Oswestry, las tres versiones solo difieren en un apartado, el apartado de actividad sexual es cambiado por trabajo/trabajo en casa ó por cambio del grado de dolor, Megan apud Davidson ${ }^{25}$ realizó un análisis para determinar la validez de los tres cuestionarios, encontrando que de preferencia se debe utilizar cualquiera de los 2 primeros.

\section{MATERIAL Y MÉTODOS}

Es un estudio de cohortes, retrospectivo, longitudinal y observacional que incluye pacientes con espondilolistesis grado I de Meyerding, tratadas con espaciador interespinoso y con seguimiento posquirúrgico a 12 meses.

El éxito del tratamiento se cuantificara en base a la escala de incapacidad de Oswestry de manera cuantitativa, además de valorarse presencia o ausencia de dolor irradiado a miembros pélvicos, como método indirecto para valorar la recuperación del paciente se tomarán en cuenta tiempo quirúrgico, días de incapacidad y días de estancia hospitalaria.

El estudio se llevó acabo en pacientes derechohabientes del servicio médico de PEMEX, tomado del expediente electrónico SIAH de enero del 2008 a diciembre del 2010, previa autorización por el comité de Bioética del H.C.S.A.E. PEMEX, se revisa el historial electrónico y radiográfico de los pacientes según los criterios de inclusión en el período de enero 2008 a diciembre 2010.

Se captura, según los criterios de inclusión, exclusión y eliminación un total de 41 pacientes de los cuales 29 pacientes cumplieron dichos criterios y fueron analizados estadísticamente.

La información de base evaluó; sexo, talla, peso, índice de masa corporal como valores demográficos a valorar, los datos prequirúrgicos que se evaluaron Oswestry prequirúrgico y dolor irradiado a miembro pélvico, los datos quirúrgicos que se evaluaron Oswestry posquirúrgico a un año, presencia o ausencia de dolor irradiado a miembro pélvico a un año, tipo de implante utilizado, días de estancia hospitalaria, días de incapacidad, tiempo quirúrgico y si el paciente fue reintervenido.

El seguimiento fue antes de la cirugía, durante su evento quirúrgico y evolución a un año, durante el estudio no se contó con ningún tipo de fuente de financiamiento.

Los criterios de inclusión que se tomaron para este estudio son espondilolistesis de un solo segmento, L4 y L5 y que no contara con cirugías de columna previas, presencia de dolor lumbar con escala de Oswestry a partir de moderado, ausencia de patología de columna agregada como: fracturas previas y tumores lumbares, uso de espaciador interespinoso como tratamiento para la espondilolistesis, haber realizado una escala de Oswestry prequirúrgica y a 12 meses de la cirugía, haber empleado tratamiento conservador de por lo menos 6 meses a base de AINEs y medidas higiénico dietéticas generales.

Los criterios de exclusión que se emplearon son la presencia de espondilolistesis de más de un segmento, que el paciente tuviera alguna cirugía previa de columna, presencia de dolor lumbar con escala de Oswestry Leve, una patología de columna lumbar agregada como fracturas y tumores.

El uso de fijación transpedicular agregada en la cirugía, que el paciente no contara con escala de Oswestry prequirúrgica y no haber recibido tratamiento conservador previo un mínimo de 6 meses.

Los criterios de eliminación son un seguimiento menor a 12 meses o que no contara con una evaluación mediante escala de Oswestry.

\section{Análisis estadístico}

Se recolectó una muestra total de 41 pacientes en el expediente electrónico SIAH de enero 2008 a diciembre 2009 y se analizó la estadística con el paquete IBM SPSS Statistics 19.

Se realizó el análisis estadístico tomando de la información recabada, frecuencia, medias y medianas, con el fin de conocer los rangos y las características demográficas de los pacientes.
Posteriormente se corre el análisis descriptivo de cada una de las variables para encontrar su significancia como factores independientes a contrastar con el objetivo final del estudio.

Después de esto, se realiza una regresión logística lineal para determinar varianzas y covarianzas que correlacionaran los factores demográficos con los factores quirúrgicos en la evolución posquirúrgica a 12 meses del paciente.

Por último, se correlacionó a través de la prueba T de Student para comparar las medias y desviaciones estándar de los grupos de datos y comparar si entre estos parámetros las muestras son estadísticamente significativas.

\section{RESULTADOS}

De los 41 pacientes que reunieron los criterios de inclusión, 9 (21\%) fueron excluidos por no contar con Oswestry posquirúrgico. 2 (4\%) pacientes fueron eliminados ya que fueron reoperados antes de cumplir el año, el motivo de la reintervención fue por falla del material.

En cuanto a frecuencias los datos relevantes se encontró que se tiene un porcentaje acumulado de $3.4 \%$ de reintervenciones del total de la muestra, el valor de Oswestry prequirúrgico fue de $3.4 \%$ leve, $55.2 \%$ moderado y $41.4 \%$ severo; mientras que el posquirúrgico fue de $79.3 \%$ leve y $20.7 \%$ moderado.

La cirugía realizada más común fue exploración y liberación con un $72.4 \%$, presentando discectomía únicamente el $27.6 \%$, los pacientes presentaron dolor irradiado a miembro pélvico derecho en el $37.9 \%$, miembro pélvico izquierdo 44.8 y a ambos miembros pélvicos en un $17.2 \%$. Se presentó dolor posquirúrgico irradiado a miembro pélvico únicamente en el $2.4 \%$ siendo que el $100 \%$ de los casos presentaron algún tipo de dolor irradiado; se utilizó espaciador tipo DIAM en $79.3 \%$ y Wallis en $20.7 \%$.

El análisis descriptivo (Tabla 1) muestra una edad ( $\mathrm{N}=29)$ con edad de $51.48 \pm 12.66$, peso $(\mathrm{N}=29)$ de $80.55+11.49$, talla $(\mathrm{N}=29)$

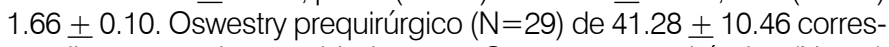
pondiente a una incapacidad severa, Oswestry posquirúrgico $(\mathrm{N}=29)$ $22.122 .1 \pm 10.77$ correspondiente a una incapacidad moderada, estancia $(\bar{N}=29)$ mínimo de 4 máximo 10 media de 6.10 días.

En cuanto al modelo de regresión lineal (Tabla 2 y 3) se utilizó Anova para análisis de Varianza utilizando como variables predictoras Oswestry prequirúrgico, edad, peso, sexo, índice de masa corporal, talla y tiempo quirúrgico y como variable dependiente Oswestry posquirúrgico con una significancia de 0.002 para este tipo de modelo.

Tabla 1. Análisis descriptivo de los datos demográficos.

\begin{tabular}{c|c|c|c|c|c}
\hline & $\mathbf{N}$ & Mínimo & Máximo & Media & Desv. standard \\
\hline Edad & 29 & 22 & 74 & 51.48 & 12.699 \\
\hline Sexo & 29 & 0 & 1 & 0.45 & 0.506 \\
\hline Peso & 29 & 60 & 100 & 80.55 & 11.49 \\
\hline Talla & 29 & 1.5 & 1.84 & 1.6607 & 0.1069 \\
\hline IMC & 29 & 23.73 & 42.22 & 29.2003 & 3.39741 \\
\hline Ox. realizada & 29 & 0 & 1 & 0.28 & 0.455 \\
\hline Tiempo Quirúrgico & 29 & 60 & 240 & 131.9 & 39.56 \\
\hline $\begin{array}{c}\text { Oswestry } \\
\text { Prequirúrgico }\end{array}$ & 29 & 19 & 60 & 41.28 & 10.464 \\
\hline $\begin{array}{c}\text { Oswestry } \\
\text { Posquirúrgico }\end{array}$ & 29 & 8 & 50 & 22.1 & 10.775 \\
\hline Incapacidad & 10 & 87 & 434 & 221.8 & 105.833 \\
\hline
\end{tabular}

Tabla 2. Regresión lineal.

\begin{tabular}{c|c|c|c}
\hline \multicolumn{3}{c}{ ANOVA $^{\mathbf{b}}$} \\
\hline \multirow{3}{*}{1} & Modelo & Suma de cuadrados & gl \\
\hline \multirow{3}{*}{1} & Regresión & 2058.475 & 7 \\
\cline { 2 - 4 } & Residual & 1192.215 & 21 \\
\cline { 2 - 4 } & Total & 3250.69 & 28 \\
\hline
\end{tabular}

a. Variables predictoras: (Constante), Osw Pre, Edad, Peso, Tiempo Qx, Sexo, IMC, Talla. b. Variable dependiente: Osw Pos. 
Se muestra que el Oswestry posquirúrgico es directamente proporcional, únicamente a la edad $(\mathrm{p}=0.098)$ y al Oswestry prequirúrgico (0.000).

Se correlacionó el Oswestry posquirúrgico contra todas las variables utilizando la $T$ de Student (Tabla 4), encontrando que solo presentan significancia estadística dolor prequirúrgico $(p=0.006)$, Oswestry prequirúrgico $(p=0.000)$, estancia hospitalaria $(p=0.001)$, reintervención $(p=0.006)$.

Se correlacionó el Oswestry prequirúrgico con los factores demográficos y el dolor prequirúrgico sin que se encontrara significancia estadística.

Tabla 3. Regresión lineal. Relación estadística de Oswestry posquirúrgico contra datos demográficos, tiempo quirúrgico y Oswestry prequirúrgico.

\begin{tabular}{c|c|c|c|c}
\hline \multicolumn{4}{c}{ Coeficientes $^{\text {a }}$} \\
\hline \multirow{2}{*}{ Modelo } & \multicolumn{2}{|c}{$\begin{array}{c}\text { Coeficientes no } \\
\text { estandarizados }\end{array}$} & $\begin{array}{c}\text { Coeficientes } \\
\text { tipificados }\end{array}$ \\
\cline { 3 - 5 } \multicolumn{2}{c|}{} & B & Error típ. & Beta \\
\hline \multirow{4}{*}{1} & (Constante) & 272.199 & 225.925 & \\
\cline { 2 - 5 } & Edad & -0.241 & 0.139 & -0.284 \\
\cline { 2 - 5 } & Sexo & -7.3 & 5.274 & -0.343 \\
\cline { 2 - 5 } & Peso & 1.388 & 1.483 & 1.48 \\
\cline { 2 - 5 } & Talla & -175.452 & 142.586 & -1.741 \\
\cline { 2 - 5 } & IMC & -3.169 & 3.757 & -0.999 \\
\cline { 2 - 5 } & Tiempo Qx & 0.054 & 0.039 & 0.197 \\
\cline { 2 - 5 } & OswPre & 0.741 & 0.153 & 0.72 \\
\hline
\end{tabular}

a. Variable dependiente: Osw Pos.

Tabla 4. Prueba T de Student. Comparación de variables para determinar su significancia estadística.

\begin{tabular}{|c|c|c|c|}
\hline Muestras Relacionadas & $\mathbf{N}$ & Correlación & Significancia \\
\hline $\begin{array}{l}\text { Edad y Oswestry } \\
\text { Posquirúrgico }\end{array}$ & 29 & -0.017 & 0.932 \\
\hline $\begin{array}{l}\text { Talla y Oswestry } \\
\text { Posquirúrgico }\end{array}$ & 29 & -0.276 & 0.147 \\
\hline $\begin{array}{l}\text { IMC y Oswestry } \\
\text { Posquirúrgico }\end{array}$ & 29 & 0.245 & 0.2 \\
\hline $\begin{array}{c}\text { Espaciador y Oswestry } \\
\text { Posquirúrgico }\end{array}$ & 29 & 0.132 & 0.496 \\
\hline $\begin{array}{c}\text { Cirugìa realizada y } \\
\text { Oswestry Posquirúrgico }\end{array}$ & 29 & 0.198 & 0.303 \\
\hline $\begin{array}{c}\text { Tiempo Quirùrgico y } \\
\text { Oswestry Posquirúrgico }\end{array}$ & 29 & 0.072 & 0.709 \\
\hline $\begin{array}{c}\text { Dolor Prequirùrgico y } \\
\text { Oswestry Posquirúrgico }\end{array}$ & 29 & 0.076 & 0.696 \\
\hline $\begin{array}{l}\text { DolorPos y Oswestry } \\
\text { Posquirúrgico }\end{array}$ & 29 & -0.498 & 0.006 \\
\hline $\begin{array}{c}\text { Incapacidad y Oswestry } \\
\text { Posquirúrgico }\end{array}$ & 10 & -0.35 & 0.322 \\
\hline $\begin{array}{c}\text { Estancia y Oswestry } \\
\text { Posquirúrgico }\end{array}$ & 29 & 0.581 & 0.001 \\
\hline $\begin{array}{c}\text { Reintervención y } \\
\text { Oswestry Posquirúrgico }\end{array}$ & 29 & -0.498 & 0.006 \\
\hline $\begin{array}{c}\text { Oswestry Prequirúrgico y } \\
\text { Oswestry Posquirúrgico } \\
\end{array}$ & 29 & 0.572 & 0.001 \\
\hline $\begin{array}{c}\text { Oswestry Prequirúrgico } \\
\text { y Edad }\end{array}$ & 29 & 0.069 & 0.724 \\
\hline $\begin{array}{c}\text { Oswestry Prequirúrgico } \\
\text { y Sexo }\end{array}$ & 29 & 0.185 & 0.337 \\
\hline $\begin{array}{c}\text { Oswestry Prequirúrgico } \\
\text { y Peso }\end{array}$ & 29 & -0.072 & 0.711 \\
\hline $\begin{array}{c}\text { Oswestry Prequirúrgico } \\
\text { y IMC }\end{array}$ & 29 & 0.261 & 0.171 \\
\hline $\begin{array}{c}\text { Oswestry Prequirúrgico y } \\
\text { Dolor Pre quirúrgico }\end{array}$ & 29 & -0.077 & 0.692 \\
\hline
\end{tabular}

\section{DISCUSIÓN}

Dentro de la literatura se ha tratado de determinar si el tratamiento quirúrgico es mejor opción que el conservador en casos de espondilositésis lumbar degenerativa; Weinstein et al. ${ }^{4}$ que el tratamiento quirúrgico presenta mejoría en cuanto al dolor, función, satisfacción a cuatro años. Dentro del estudio realizado se trató de eliminar la controversia en cuanto a que funciona mejor si el tratamiento quirúrgico o no quirúrgico, ya que solo se incluyeron pacientes con 6 meses de tratamiento conservador. No se intentó evaluar la efectividad del tratamiento conservador ya que la falta de adherencia al tratamiento y la variedad de tratamientos utilizados variaban en gran medida.

En el estudio se encontró una mejoría estadísticamente significativa para el Oswestry posquirúrgico $(p=0.000)$ teniendo una media $22.1 \%$ a un año del evento quirúrgico. No se encontró un estudio similar para comparar directamente el resultado del estudio ya sea con fusión lumbar o con espaciadores dinámicos ya que generalmente involucran otros tratamientos. Sin embargo se encuentra reportado por Weintstein et al. ${ }^{4}$ un Oswestry con una media de $21.9 \%$ aunque se realizó laminectomía con o sin fusión lumbar. Ha sido sugerido por Birkmeyer et al. ${ }^{26}$ que una diferencia de 10 puntos en la escala de Oswestry representa una diferencia clínica significativa. Durante el estudio se encontró un mejoría media de 19.18. Esto puede ser comparable con lo reportado por Ghogawala et al. ${ }^{27}$ quien reporta en mejoría de 19.5 en el tratamiento de espondilolistesis grado I tratada con descompresión con y sin fusión

Si bien, la fusión lumbar sigue siendo el standard a seguir, Deyo et al. ${ }^{28}$ reporta mayor índice de complicaciones, siendo la incidencia de raíz nerviosa hasta un $60 \%$ mas frecuente en paciente con descompresión, sin fusión. ${ }^{29}$ En contraparte, en un metanálisis de la literatura, se encontró que los pacientes tratados únicamente con descompresión presentaban un resultado satisfactorio en un $69 \%$, mientras que los que además se les realizó fusión presentaron $90 \%$ de satisfacción. ${ }^{30}$ Teniendo en cuenta estos factores, la posibilidad de tratar la espondilolistesis de manera dinámica tendría un valor agregado, ya que tendría menos complicaciones con un satisfacción mayor del paciente. Aunque es necesario dar seguimiento a mas de un año y aumentar el numero de pacientes para poder comprobar dicha hipótesis.

Dentro de los datos importantes que el estudio de igual manera ofreció, fue que la estancia hospitalaria, así como la edad son las únicas otras dos variables estadísticamente significativas del estudio, si bien podemos correlacionar directamente con la evolución posquirúrgica, es importante mencionar que en lo que respecta a la estancia hospitalaria total no puede evaluarse como un factor único y aislado, esto se debe a que los factores por los cuales un paciente debe permanecer hospitalizado no dependen únicamente del acto quirúrgico o de las consecuencias del mismo por lo que tendría que ser estudiado con un mayor número de casos y correlacionando dichas variables de manera independiente.

Dentro de los parámetros importantes a determinar en la institución, es si este tratamiento tiene algún tipo de efecto sobre los días de incapacidad del trabajador, se obtuvieron resultados con una media de 221 días de incapacidad, si bien no se cuenta con un parámetro para comparar, como lo serían los días de incapacidad en trabajadores a los que se les realiza la fijación de L4-L5, se puede decir que se encuentra dentro de un parámetro aceptable para el tipo de patología ya que la fusión lumbar puede tardar de 3 a 4 meses en reincorporarse a la vida laboral, también es importante mencionar que solo el 34\% de los pacientes eran trabajadores activos por lo que sería importante aumentar la muestra.

\section{CONCLUSIONES}

La evolución de la incapacidad funcional a un año de la cirugía no se ve afectada de manera directa por las variables dependientes

La evolución de la incapacidad funcional a un año se ve afectada 
de manera directa por la incapacidad previa a la cirugía así como por los días de estancia hospitalaria, es decir a mayor índice de incapacidad previo a la cirugía menor será el índice de incapacidad posterior a la misma, a más días de hospitalización mayor será el índice de incapacidad posterior a la cirugía.

El tratamiento con espaciador interespinoso presenta un bajo índice de reintervención y por lo menos a un año presenta mejoría significativa en el índice de incapacidad.

Debido a que la muestra arroja una edad relativamente joven (51 años) y que podemos asumir que dichos pacientes mantienen demandas funcionales altas, es importante que el estudio se siga por lo menos a 5 años para determinar vida media a 5 años del implante así como incapacidad.

Se puede decir que el espaciador interespinoso es una opción relativamente segura y efectiva para el tratamiento de la espondilolistesis degenerativa Grado I de Mayerding, aunque aún haya que determinar su seguridad y eficacia evaluado contra un periodo de tiempo más prolongado.

Todos los autores declaran no existir ningún potencial conflicto de intereses referente a este artículo.

\section{REFERENCIAS}

1. Newman $\mathrm{PH}$, Sonte $\mathrm{KH}$. The etiology of spondylolisthesis. J Bone Joint Surg Br. 1963:45:39-59.

2. Sanderson PL, Fraser RD. The influence of pregnancy on the development of degenerative spondylolisthesis. J Bone Joint Surg Br. 1996;78(6):951-4.

3. Fredrickson BE, Baker D, McHolick WJ, Yuan HA, Lubicky JP. The natural history of spondylolysis and spondylolisthesis. J Bone Joint Surg Am. 1984;66(5):699-707.

4. Weinstein JN, Lurie JD, Tosteson TD, Hanscom B, Tosteson AN, Blood EA, et al. Surgical versus nonsurgical treatment for lumbar degenerative spondylolisthesis. N Engl J Med. 2007:356(22):2257-70.

5. Fischgrund JS, Mackay M, Herkowitz HN, Brower R, Montgomery DM, Kurz LT. 1997 Volvo Award winner in clinical studies. Degenerative lumbar spondylolisthesis with spinal stenosis: a prospective, randomized study comparing decompressive laminectomy and arthrodesis with and without spinal instrumentation. Spine (Phila Pa 1976). 1997;22(24):2807-12.

6. Herkowitz HN, Kurz LT. Degenerative lumbar spondylolisthesis with spinal stenosis. A prospective study comparing decompression with decompression and intertransverse process arthrodesis. J Bone Joint Surg Am. 1991;73(6):802-8.

7. Hu SS, Tribus CB, Diab M, Ghanayem AJ. Spondylolisthesis and spondylolysis. J Bone Joint Surg Am. 2008:90(3):656-71.

8. Meyerding HW. Spondylolisthesis. J Bone Joint Surg. 1931;13:39-48.

9. Zigler J, Delamarter R, Spivak JM, Linovitz RJ, Danielson GO 3rd, HaiderTT, et al. Results of the prospective, randomized, multicenter Food and Drug Administration investigational device exemption study of the ProDisc-L total disc replacement versus circumferential fusion for the treatment of 1-level degenerative disc disease. Spine (Phila Pa 1976). 2007;32(11):1155-62.

10. Kondrashov DG, Hannibal M, Hsu KY, Zucherman JF. Interspinous process decompression with the X-STOP device for lumbar spinal stenosis: a 4-year follow-up study. J Spinal Disord Tech. 2006;19(5):323-7.

11. Nockels RP. Dynamic stabilization in the surgical management of painful lumbar spinal disorders. Spine (Phila Pa 1976). 2005;30(Suppl 16):S68-72

12. Mulholland RC, Sengupta DK. Rationale, principles and experimental evaluation of the concept of soft stabilization. Eur Spine J. 2002:11(Suppl 2):S198-205.

13. McNally DS, Shackleford IM, Goodship AE, Mulholland RC. In vivo stress measurement can predict pain on discography. Spine (Phila Pa 1976). 1996;21(22):2580-7.

14. Sengupta DK. Dynamic stabilization devices in the treatment of low back pain. Orthop Clin North Am. 2004;35(1):43-56.

15. Lindsey DP, Swanson KE, Fuchs P, Hsu KY, Zucherman JF, Yerby SA. The effects of an interspinous implant on the kinematics of the instrumented and adjacent levels in the

lumbar spine. Spine (Phila Pa 1976). 2003;28(19):2192-7.

16. Minns RJ, Walsh WK. Preliminary design and experimental studies of a novel soft implant for correcting sagittal plane instability in the lumbar spine. Spine (Phila Pa 1976). 1997;22(16):1819-25.

17. Christie SD, Song JK, Fessler RG. Dynamic interspinous process technology. Spine (Phila Pa 1976). 2005:30(Suppl 16):S73-8

18. Taylor J, Pupin P, Delajoux S, Palmer S. Device for intervertebral assisted motion: technique and initial results. Neurosurg Focus. 2007;22(1):E6.

19. Bellini CM, Galbusera F Raimondi MT, Mineo GV, Brayda-Bruno M. Biomechanics of the lumbar spine after dynamic stabilization. J Spinal Disord Tech. 2007;20(6):423-9.

20. Rohlmann A, Burra NK, Zander T, Bergmann G. Comparison of the effects of bilateral posterior dynamic and rigid fixation devices on the loads in the lumbar spine: a finite element analysis. Eur Spine J. 2007:16(8):1223-31.

21. Ahn YH, Chen WM, Lee KY, Park KW, Lee SJ. Comparison of the load-sharing characteristics between pedicle-based dynamic and rigid rod devices. Biomed Mater. 2008:3(4):44-101.

22. Sénégas J. Mechanical supplementation by non-rigid fixation in degenerative intervertebral lumbar segments: the Wallis system. Eur Spine J. 2002;11 (Suppl 2):S164-9.

23. Fairbank JC, Couper J, Davies JB, O'Brien JP. The Oswestry low back pain disability questionnaire. Physiotherapy. 1980;66(8):271-3.

24. Fairbank JC, Pynsent PB. The Oswestry Disability Index. Spine (Phila Pa 1976). 2000;25(22):2940-52.

25. Davidson M. Rasch analysis of three versions of the Oswestry Disability Questionnaire. Man Ther. 2008;13(3):222-31.

26. Birkmeyer NJ, Weinstein JN, Tosteson AN, Tosteson TD, Skinner JS, Lurie JD, et al. Design of the Spine Patient outcomes Research Trial (SPORT). Spine (Phila Pa 1976). 2002;27(12):1361-72.

27. Ghogawala Z, Benzel EC, Amin-Hanjani S, Barker FG 2nd, Harrington JF, Magge SN, et al. Prospective outcomes evaluation after decompression with or without instrumented fusion for lumbar stenosis and degenerative Grade I spondylolisthesis. J Neurosurg Spine. 2004; 1(3):267-72

28. Deyo RA, Ciol MA, Cherkin DC, Loeser JD, Bigos SJ. Lumbar spinal fusion. A cohort study of complications, reoperations, and resource use in the Medicare population. Spine (Phila Pa 1976). 1993;18(11):1463-70.

29. Weir B, de Leo R. Lumbar stenosis: analysis of factors affecting outcome in 81 surgical cases. Can J Neurol Sci. 1981:8(4):295-8.

30. Mardjetko SM, Connolly PJ, Shott S. Degenerative lumbar spondylolisthesis. A meta-analysis of literature 1970-1993. Spine (Phila Pa 1976). 1994;19(Suppl 20):2256S-65S. 